\title{
The Current Role of Induced Endometrial Trauma (Endometrial Scratch) in Women Undergoing Infertility Treatment
}

\author{
Mostafa Metwally, FRCOG, MD ${ }^{1}$ Stephen Walters, $\mathrm{PhD}^{2}$ Robin Chatters, $\mathrm{BSc}^{2}$ \\ ${ }^{1}$ The Academic Unit of Reproductive and Developmental Medicine, \\ The University of Sheffield and Sheffield Teaching Hospitals, \\ Sheffield, United Kingdom \\ ${ }^{2}$ The Clinical Trials Research Unit, The University of Sheffield,

\begin{abstract}
Address for correspondence Mostafa Metwally, FRCOG, MD, The Academic Unit of Reproductive and Developmental Medicine, The University of Sheffield and Sheffield Teaching Hospitals, Sheffield, United Kingdom (e-mail: m.metwally@sheffield.ac.uk).
\end{abstract} Sheffield, United Kingdom

Semin Reprod Med 2021;39:e1-e4

\begin{abstract}
Keywords

- IVF

- endometrial scratch

- endometrial trauma

- infertility add on

Induced endometrial trauma, otherwise known as endometrial scratch is a simple technique that has been rapidly adopted into clinical practice, mainly for women having IVF treatment, in an attempt to increase pregnancy rates. The introduction of endometrial scratch followed early reports of improved clinical pregnancy rates in women with repetitive implantation failure after having the procedure and follows on from evidence from animal models in the early $20^{\text {th }}$ century suggesting that mechanical trauma to the endometrium can induce decidual changes. Due to the ease and low cost of the procedure, it has been rapidly adopted as an add-on to fertility treatments, in many cases where evidence is still lacking. Despite the initial publication of a large number of studies that demonstrated encouraging improvements in pregnancy rates in women who underwent this procedure, these studies were mainly limited by the small sample sizes and heterogeneity of their study populations, leading to limited validity of the evidence provided by these studies. More recently, three large randomized controlled studies have been published that paint a different picture regarding the value of this procedure. This article explores the evolution of the evidence and the current state of endometrial scratch as an adjuvant therapy for women undergoing IVF treatment.
\end{abstract}

The use of local endometrial trauma known as Endometrial Scratch (ES) to improve implantation rates in women undergoing assisted conception was first described in $2003^{1}$ and was initially sparked by a paper investigating the pattern of endometrial expression of gap junction proteins. Repetitive endometrial sampling was performed for 12 patients undergoing in vitro fertilization (IVF) and recurrent implantation failure (RIF), interestingly 11 of these patients became pregnant. $^{2}$ The concept of using a mechanical stimulus to cause decidual endometrial changes however goes back many decades where Leo Loeb (1869-1959), an experimen- tal pathologist and physician had demonstrated that "transitory neoplasms of the uterine mucosa, deciduomas and placentomas, could be caused mainly by two factors, first a sensitization of the uterine mucosa by the hormone of the corpus luteum, followed secondarily by the application of mechanical stimuli, such as a wound or the introduction of a foreign body into the uterine lumen"3. Over the last two decades the use of the controlled endometrial trauma has gained significant popularity as an adjuvant treatment that for nearly every group of women with fertility problems in attempt to improve the implantation potential of the
Issue Theme Adjuvant Therapies in ART; Guest Editors, Thomas Tang, MD, MRCOG, Nikoletta Panagiotopoulou, MRCOG, MSc, MD, and Ephia Yasmin, $M D, M R C O G$ (c) 2021. Thieme. All rights reserved. Thieme Medical Publishers, Inc., 333 Seventh Avenue, 18th Floor, New York, NY 10001, USA
DOI https://doi.org/ 10.1055/s-0041-1739162. ISSN 1526-8004. 
endometrium. While some medical procedures are often slow to be accepted widely into medical practice, endometrial scratch is one particular situation where an interventional procedure has been rushed into clinical practice at a speed which far exceeds the time necessary for generation of good quality evidence. The procedure has become so popular that a recent survey ${ }^{4}$ showed that $83 \%$ of surveyed clinicians recommended an endometrial scratch. $77 \%$ for recommended it for women undergoing IVF and ICSI, 3.6\% for women suffering from recurrent miscarriage and yet another $3.6 \%$ even recommended it for women trying naturally or with intrauterine insemination. Amongst those performing it for IVF patients, the majority recommended it for women with recurrent implantation failure (92\%) but some even recommended it for all women undergoing IVF (4\%). Given how common this procedure is, it is of extreme importance that we objectively evaluate the evidence for its clinical use. This article will aim to summarize the current state of the evidence for the use of endometrial scratch in clinical practice in different infertile groups

\section{Proposed Mechanisms by which Endometrial Scratch May Improve The Endometrial Implantation Potential?}

Proposed mechanisms are discussed in more detail elsewhere in this edition (Adjuvant therapy in ART, Part One), but include the potential release of inflammatory mediators including uterine natural killer cells, leukemia inhibitory factor and interleukin $15,{ }^{5}$ macrophages and dendritic cells, tumor necrosis factor- $\alpha$, interleukin-15, growth-regulated oncogene- $\alpha$ and macrophage inflammatory protein $1 \mathrm{~B}^{6}{ }^{6} \mathrm{ES}$ has also been shown to cause the modulation of several endometrial genes that may be involved in membrane stability during the process of implantation such as bladder transmembranal protein (UPIb) and adipose differentiationrelated protein and mucin $1^{7}$ and through enhancement of endometrial angiogenesis through an effect on matrix metalloproteinase-3 (MMP-3), plasminogen activator inhibitor-1 (PAI-1), insulin-like growth factor binding protein 1 (IGFBP$1)$, and IL- $1 \alpha^{8}$

\section{Endometrial scratch for Women Undergoing IVF}

Since the earliest study by Barash et al, ${ }^{1}$ several studies focusing mainly on women with recurrent implantation failure demonstrated a significant increase in pregnancy rates by almost double. ${ }^{9-11}$ However, conflicting evidence was provided by at least one randomized controlled study of 156 participants $^{12}$ that suggested that the procedure was harmful with a significant reduction in pregnancy rates [OR of clinical pregnancy rate of $0.30(0.14,0.63) p=0.002] .^{8}$ Notably, this trial performed the ES procedure at the time of oocyte retrieval and not in the month prior to the IVF cycle. Another RCT of 132 embryo transfer cycles randomized to receive an endometrial scratch procedure or not, ${ }^{13}$ was stopped prematurely after an unplanned interim analysis showed a trend toward a lower clinical pregnancy rates in the endometrial scratch arm (23.5\%) compared with the control $\operatorname{arm}$ (35.9\%), (hazard ratio $=0.43 ; 95 \% \mathrm{CI}, 0.18-1.02$; $p=0.0568$ ). However, this study was underpowered and more recent studies with larger sample size have not shown evidence of harm. ${ }^{14}$

Overall however the majority of earlier evidence pointed to an improvement in fertility outcomes which is reflected in the 2015 Cochrane review that suggested a significant improvement in live birth rates in women with recurrent implantation failure undergoing endometrial scratch and classed the evidence as moderate quality. ${ }^{15}$

\section{The Turning Tides}

There have since been several key studies that should be pointed out. The first study by Yeung et al, (2014) was conducted in an unselected population of women undergoing IVF, of whom nearly $70 \%$ were having their first IVF cycle. ${ }^{16}$ No significant differences were seen in the unselected population in ongoing pregnancy rates, miscarriage rates, clinical pregnancy rates, implantation rates and multiple pregnancy rates. The authors performed a subgroup analysis in women undergoing their first embryo transfer $(N=209)$ and similarly found no difference in ongoing pregnancy rate while they noted a significantly lower pregnancy rate in control women who had had previous treatment failure $(N=91)$. However, the study was not powered for these subgroup analyses and therefore no reliable conclusions can be drawn. Also, a mixture of protocols was used and there were no restrictions regarding age or day of embryo transfer with most patients receiving two embryo transfers. $^{16}$

The second study by Lensen et al, (2019), included 1364 women who were randomized to endometrial scratch or no intervention and found that endometrial scratch did not result in an increase in live birth rate. ${ }^{14}$ Although this study was somewhat interpreted as conclusive evidence against the use of endometrial scratch, ${ }^{17}$ a careful examination of the study population leads to a different conclusion.

The study included different subgroups undergoing IVF to maintain a pragmatic approach but in doing so also introduced potential methodological problems. First, the title implied that the findings pertain to all women undergoing IVF when this is not the case. The study combined a mixture of patients with different prognostic potential. The study had two main subgroups, the first is women with recurrent implantation failure and the second is women who have had a maximum of one previous cycle. These are two very different populations with different prognostic outcomes. The study therefore did not include a specific group of women having their first IVF cycle and was not powered specifically for this group. Furthermore, there is no report on the live birth rate for this group. The findings therefore cannot be generalized to include woman having their first IVF cycle.

Patients undergoing IVF represent a large heterogeneous group of women and the results of any IVF study is subject to 
several inherent causes for bias that lie within the underlying pathology and the characteristics of the studied population. It is therefore of upmost importance to target the clinical question to a very specific and homogenous group of participants. In this case, the heterogeneity is further increased and the power is further compromised by including both fresh and frozen IVF cycles. Fresh and frozen embryo transfers are different populations and it is not ideal to combine them within one analysis. The dynamics of the endometrium are different in fresh and frozen cycle and combining the two introduces further cause for bias. There is currently a large UK national randomized control study (the E-Freeze study) which is looking at the possible increased implantation potential in women having frozen embryo replacement cycles compared with fresh cycles and until the results are published it is uncertain how the combination of these two groups could have influenced the results. ${ }^{18}$

Furthermore, the study did not exclude potential clinical confounders where the endometrium may have been compromised. For example, $10.9 \%$ of women in the scratch group and $12.8 \%$ of women in the control group had ovulatory disorders. Although the nature of the ovulatory disorder is not specified, polycystic ovarian syndrome can be associated with hyperandrogenism and an adverse effect on the endometrium. Furthermore, patients with endometriosis were included and the use of an ultra-long protocol for ovarian stimulation suggests that some women may have had severe endometriosis. Severe endometriosis can be associated with an adverse effect on the endometrium and therefore it would have been ideal to exclude these two groups from the analysis or perform a sensitivity analysis. It is also noted that $7.2 \%$ of women with the intervention and $4.2 \%$ of women in the control group received a short (flare) protocol which is often used in women who have a low ovarian reserve and this may again have influenced results. Similar potential causes for bias include the variability in the number of embryos transferred, the day of embryo transfer and the phase of the cycle where the scratch was performed. The conclusions of this study therefore should be limited mainly to women having recurrent implantation failure.

Regarding women undergoing first time IVF treatment. Our recently published randomized controlled study ${ }^{19}$ was powered only to this particular group and attempted to minimize bias and heterogeneity by including only women predicted to have a good response with no significant pathologies that may affect the endometrium and women expected to have a single blastocyst transfer. Similar to the findings of Lensen ${ }^{14}$ however, we found no evidence of improvement in live birth rates with the use of endometrial scratch [ES $(n=523) 37.1 \%$, Control $(n=525) 38.6 \%$; $95 \%$ C.I. $-4.4 \%$ to $7.4 \%, p=0.621]$.

Addressing the middle part of the spectrum, is the recently published study from the Netherlands that focused on women who had only one previously unsuccessful IVF cy$\mathrm{cle}^{20}$ and again found no evidence of a significant improvement in live birth rate [ES $(n=465) 23.7 \%$, Control $(n=461)$ 19.1\%; R.R. 95\% C.I. 096-1.59]. Neither studies demonstrated any increased in adverse effects and miscarriage rates.
Although this study recommends further research into endometrial scratch for the IVF population, when viewed in the context of the two other studies, ${ }^{14,19}$ it is difficult to support this recommendation. All three studies have examined different populations yet have produced similar results. It seems therefore that the use of endometrial scratch should no longer be offered for any population of women undergoing IVF.

\section{Endometrial Scratch for Women Undergoing Other Fertility Treatments}

The potential role of endometrial scratch has also been examined in women undergoing other fertility treatments such as intrauterine insemination (IUI) with conflicting and inconclusive results. The two main problems with the current evidence is that the studies have been relatively small and therefore potentially underpowered and inconclusive $e^{21-24}$ and consequently the overall quality of the evidence has been poor. At least two meta-analyses have systematically analyzed the evidence. The more recent meta-analysis included eight trials with a total of 1,871 IUI cycles and found that the clinical and ongoing clinical pregnancy rates were more than doubled in women having the endometrial scratch prior to IUI, but this was limited to those having the procedure in the early follicular phase of the treatment cycle rather than in the preceding cycle. ${ }^{25}$ The earlier Cochrane review included nine trials with a total of 1512 women. Although the study found similar findings i.e., a potential improvement in clinical pregnancy rates with endometrial scratch, the quality of the evidence was classed as very poor and therefore the results were found to be inconclusive. ${ }^{4}$ Similarly, the evidence for the use of endometrial scratch to improve pregnancy rates in women with unexplained infertility or those trying to conceive naturally is far from conclusive and the evidence is poor. ${ }^{4}$

\section{Conclusion}

Endometrial scratch is an example of an add-on treatment that has been rapidly adopted into the field of reproductive medicine without the backing of good evidence. It is probably the simplicity of the technique that led to its rapid adoption into the field and was initially supported by a plethora of studies that were limited by inherent bias as a result of small sample sizes and heterogeneity. With the gradual emergence of newer studies that have been large enough to attain adequate power and homogenous enough to address some of the inherent heterogeneity in infertility populations, there is now little doubt that this technique offers no benefit for women undergoing IVF treatment. While evidence for other infertility populations is awaited, the evidence that we now have makes it difficult for any clinician to continue to offer this treatment in the hope that it will increase the chances of a live birth.

Conflict of Interest

None. 


\section{References}

1 Barash A, Dekel N, Fieldust S, Segal I, Schechtman E, Granot I. Local injury to the endometrium doubles the incidence of successful pregnancies in patients undergoing in vitro fertilization. Fertil Steril 2003;79(06):1317-1322

2 Granot I, Dekel N, Bechor E, Segal I, Fieldust S, Barash A. Temporal analysis of connexin43 protein and gene expression throughout the menstrual cycle in human endometrium. Fertil Steril 2000;73 (02):381-386

3 Goodpasture EW. Leo Loeb, September 21, 1869-December 28, 1959. New York: National Academy of Sciences; 1961

4 Lensen SF, Manders M, Nastri CO, et al. Endometrial injury for pregnancy following sexual intercourse or intrauterine insemination. Cochrane Database Syst Rev 2016;CD011424(06): CD011424. Doi: 10.1002/14651858.CD011424.pub2

5 Tuckerman E, Mariee N, Prakash A, Li TC, Laird S. Uterine natural killer cells in peri-implantation endometrium from women with repeated implantation failure after IVF. J Reprod Immunol 2010; 87(1-2):60-66

6 Gnainsky Y, Granot I, Aldo PB, et al. Local injury of the endometrium induces an inflammatory response that promotes successful implantation. Fertil Steril 2010;94(06):2030-2036

7 Kalma Y, Granot I, Gnainsky Y, et al. Endometrial biopsy-induced gene modulation: first evidence for the expression of bladdertransmembranal uroplakin Ib in human endometrium. Fertil Steril 2009;91:1042-1049, 1049 e1041-1049

8 Yang JH, Chen $\mathrm{CD}$, Chou $\mathrm{CH}$, et al. Intentional endometrial injury increases embryo implantation potentials through enhanced endometrial angiogenesis. Biol Reprod 2019;100(02):381-389

9 El-Toukhy T, Sunkara S, Khalaf Y. Local endometrial injury and IVF outcome: a systematic review and meta-analysis. Reprod Biomed Online 2012;25(04):345-354

10 Raziel A, Schachter M, Strassburger D, Bern O, Ron-El R, Friedler S. Favorable influence of local injury to the endometrium in intracytoplasmic sperm injection patients with high-order implantation failure. Fertil Steril 2007;87(01):198-201

11 Huang SY, Wang CJ, Soong YK, et al. Site-specific endometrial injury improves implantation and pregnancy in patients with repeated implantation failures. Reprod Biol Endocrinol 2011;9:140

12 Karimzade MA, Oskouian H, Ahmadi S, Oskouian L. Local injury to the endometrium on the day of oocyte retrieval has a negative impact on implantation in assisted reproductive cycles: a randomized controlled trial. Arch Gynecol Obstet 2010;281(03): 499-503

13 Frantz S, Parinaud J, Kret M, et al. Decrease in pregnancy rate after endometrial scratch in women undergoing a first or second in vitro fertilization. A multicenter randomized controlled trial. Hum Reprod 2019;34(01):92-99
14 Lensen S, Osavlyuk D, Armstrong S, et al. A Randomized Trial of Endometrial Scratching before In Vitro Fertilization. N Engl J Med 2019;380(04):325-334

15 Nastri CO, Lensen SF, Gibreel A, et al. Endometrial injury in women undergoing assisted reproductive techniques. Cochrane Database Syst Rev 2015;CD009517(03):CD009517. Doi: 10.1002/14651858. CD009517.pub3

16 Yeung TW, Chai J, Li RH, Lee VC, Ho PC, Ng EH. The effect of endometrial injury on ongoing pregnancy rate in unselected subfertile women undergoing in vitro fertilization: a randomized controlled trial. Hum Reprod 2014;29(11):2474-2481

17 Mol BW, Barnhart KT. Scratching the Endometrium in In Vitro Fertilization - Time to Stop. N Engl J Med 2019;380(04):391-392

18 Maheshwari A, Bhattacharya S, Bowler U, et al. Study protocol: Efreeze - freezing of embryos in assisted conception: a randomised controlled trial evaluating the clinical and cost effectiveness of a policy of freezing embryos followed by thawed frozen embryo transfer compared with a policy of fresh embryo transfer, in women undergoing in vitro fertilisation. Reprod Health 2019; 16(01):81

19 Metwally M, Chatters R, Dimairo M, et al. A randomised controlled trial to assess the clinical effectiveness and safety of the endometrial scratch procedure prior to first-time IVF, with or without ICSI. Hum Reprod 2021;36(07):1841-1853

20 van Hoogenhuijze NE, Mol F, Laven JSE, et al. Endometrial scratching in women with one failed IVF/ICSI cycle-outcomes of a randomised controlled trial (SCRaTCH). Hum Reprod 2021;36 (01):87-98

21 Goel T, Mahey R, Bhatla N, Kalaivani M, Pant S, Kriplani A. Pregnancy after endometrial scratching in infertile couples undergoing ovulation induction and intrauterine insemination cycles-a randomized controlled trial. J Assist Reprod Genet 2017;34(08):1051-1058

22 Senocak GC, Yapca OE, Borekci B. Comparison of pregnancy rates between patients with and without local endometrial scratching before intrauterine insemination. J Gynecol Obstet Hum Reprod 2017;46(09):687-690

23 Zarei A, Alborzi S, Dadras N, Azadi G. The effects of endometrial injury on intrauterine insemination outcome: A randomized clinical trial. Iran J Reprod Med 2014;12(09):649-652

24 Bahaa Eldin AM, Abdelmaabud KH, Laban M, et al. Endometrial Injury May Increase the Pregnancy Rate in Patients Undergoing Intrauterine Insemination: An Interventional Randomized Clinical Trial. Reprod Sci 2016;23(10):1326-1331

25 Vitagliano A, Noventa M, Saccone G, et al. Endometrial scratch injury before intrauterine insemination: is it time to re-evaluate its value? Evidence from a systematic review and meta-analysis of randomized controlled trials. Fertil Steril 2018;109(01):84-96.e4 